\title{
Fecal Microbiota of Toxigenic Clostridioides difficile-Associated Diarrhea
}

\author{
Marta Hernández ${ }^{1,2}$, Mónica de Frutos ${ }^{3}$, David Rodríguez-Lázaro², Luis López-Urrutia ${ }^{3}$, \\ Narciso M. Quijada ${ }^{1,2}$ and Jose María Eiros ${ }^{3 *}$ \\ ${ }^{1}$ Laboratorio de Biología Molecular y Microbiología, Instituto Tecnológico Agrario de Castilla y León, Valladolid, Spain, ${ }^{2}$ Área \\ de Microbiología, Departamento de Biotecnología y Ciencia de los Alimentos, Universidad de Burgos, Burgos, Spain, \\ ${ }^{3}$ Hospital Universitario Río Hortega, Valladolid, Spain
}

\section{OPEN ACCESS}

Edited by:

Meina Neumann-Schaal,

German Collection of Microorganisms and Cell Cultures GmbH (DSMZ),

Germany

Reviewed by:

Rosa Del Campo, Instituto Ramón y Cajal

de Investigación Sanitaria, Spain Alexander G. Haslberger, Universität Wien, Austria

${ }^{*}$ Correspondence:

Jose María Eiros

jmeirosbouza@gmail.com

Specialty section:

This article was submitted to Infectious Diseases,

a section of the journal

Frontiers in Microbiology

Received: 27 September 2018

Accepted: 21 December 2018

Published: 14 January 2019

Citation:

Hernández M, de Frutos $M$ Rodríguez-Lázaro D, López-Urrutia L, Quijada NM and Eiros JM (2019) Fecal Microbiota of Toxigenic Clostridioides difficile-Associated Diarrhea. Front. Microbiol. 9:3331. doi: 10.3389/fmicb.2018.03331
Clostridioides difficile infection (CDI) is currently one of the most important causes of infectious diarrhea in developed countries and the main cause in healthcare settings. Here, we characterized the gut microbiota from the feces of 57 patients with diarrhea from nosocomial and community-acquired CDI. We performed an ecological analysis by high-throughput sequencing of the V3-V4 region of $16 \mathrm{~S}$ rRNA amplicons and evaluated the association of the various ecological profiles with CDI risk factors. Among all samples Bacteroidaceae 31.01\%, Enterobacteriaceae 9.82\%, Lachnospiraceae 9.33\%, Tannerellaceae 6,16\%, and Ruminococcaceae 5.64\%, were the most abundant families. A reduced abundance of Bacteroides was associated with a poor CDI prognosis, with severe diarrhea and a high incidence of recurrence. This reduction was associated with a weakened host immune system and previous aggressive antibiotherapy. Peptostreptococcaceae family was $1.56 \%$ overall and within the family the only identified member was the genus Clostridioides, positively correlated with the presence of Akkermansia that may be predictive of the presence of a CDI. Finally, a relevant aspect that must be considered in clinical practice is the misdiagnosis of CDI, as patients with a stool sample that tests positive for $C$. difficile are usually diagnosed with $\mathrm{CDI}$ and subsequently treated as such. However, co-infection with other pathogenic agents often plays an important role in the development of diarrhea, and must be considered when prescribing antibiotic treatment.

Keywords: CDI, Clostridioides difficile, diarrhea, microbiota, bacterial, 16S rRNA

\section{INTRODUCTION}

The Gram-positive, spore-forming anaerobe, Clostridioides difficile (formerly Clostridium difficile and Peptoclostridium difficile; Yutin and Galperin, 2013) is an asymptomatic component of the healthy intestinal microbiota of approximately $2-7 \%$ of healthy human adults and up to $70 \%$ of healthy newborns (McFarland et al., 1989; Lees et al., 2016). However, certain C. difficile strains have pathogenic potential mediated by two exotoxins: toxin A (TcdA) and toxin B (TcdB), encoded by the $t c d \mathrm{~A}$ and $t c d \mathrm{~B}$ genes within the pathogenicity loci (PaLoc). In addition, some $C$. difficile strains may also produce a binary toxin, called $C$. difficile transferase (CDT), with a potential role in the pathogenesis of the bacterium (Di Bella et al., 2016). C. difficile infection (CDI) is thus a 
toxin-mediated disease of the colon, with clinical symptoms that range from mild or self-limiting diarrhea to pseudomembranous colitis and life-threatening fulminant colitis (Leffler and Lamont, 2015; Smits et al., 2016).

Clostridium difficile infection is currently one of the most important causes of infectious diarrhea in developed countries and the main cause in healthcare settings (Lagier, 2016). The rate of recurrence and mortality has been increasing since 2002, associated with severe infections produced by the emergence of the hyper-virulent ribotype 027 (ST1) strain. The burden of CDI has increased mainly in the United States and Europe. It is estimated that nearly 500,000 illnesses and 15,000 deaths are caused by CDI every year in the United States ${ }^{1}$, whereas the annual incidence in the EU was estimated to be 123,997 cases in 2011-2012 $2^{2}$, with mortality rates of 3-30\% (Hensgens et al., 2013). Remarkably, although 125 ribotypes have being found in Europe, the hyper-virulent ribotype 027 is the most prevalent (19\%) (Davies et al., 2016). In addition, the incidence of CDI is increasing and may be highly underestimated (Alcalá et al., 2015), $23 \%$ of cases may be undiagnosed, equivalent to approximately 40,000 missed CDI diagnoses per year in 482 participating hospitals in 20 European countries (Davies et al., 2014). Other relevant factors associated with the emergence of CDI have been the introduction of $C$. difficile strains resistant to multiple antibiotics, including metronidazole, as well as genomic plasticity and the potential to transfer resistance genes, as approximately $11 \%$ of the C. difficile genome consists of mobile genetic elements (Sebaihia et al., 2006).

Clostridium difficile infection appears particularly after antibiotic chemotherapy or prolonged periods of hospitalization, which causes disruption and dysbiosis of the endogenous intestinal microbiota and facilitates the proliferation of toxigenic C. difficile in the gut. Fecal-oral transmission from other patients or animals, which represent potential reservoirs of C. difficile, also plays an important role in CDI epidemiology (Rupnik, 2007). There are also other risk factors that trigger CDI, including comorbidities, surgical and non-surgical gastrointestinal procedures, admission to an intensive care unit (ICU), an immunocompromised status, and advanced age (>65 years) (Knight et al., 2015). CDI has a high relapse rate due to reactivation or reinfection, making it difficult to completely resolve. However, fecal microbiota transplantation appears to be a promising treatment for recurrent CDI (Shankar et al., 2014; Juul et al., 2018).

It is evident that modification of the gut microbiota can play a relevant role in the development of CDI. The identification of microbial markers that can predict disease severity or chronicity could help in the treatment of patients. Here, we characterized the gut microbiota from the feces of 57 patients with diarrhea from nosocomial or community-acquired CDI. The study consisted of an ecological analysis by high-throughput sequencing of the $\mathrm{V} 3-\mathrm{V} 4$ region of the $16 \mathrm{~S}$ rRNA amplicons and evaluation of the association of the various ecological profiles with CDI risk factors.

\footnotetext{
${ }^{1}$ www.cdc.gov/media/releases/2015/p0225-clostridium-difficile.html

${ }^{2}$ https://ecdc.europa.eu/sites/portal/files/media/en/publications/Publications/ healthcare-associated-infections-antimicrobial-use-PPS.pdf
}

\section{MATERIALS AND METHODS}

\section{Sampling and Detection of Toxins}

This study was conducted at the Hospital Universitario "Rio Hortega" (Valladolid, Castilla y León, Spain) in accordance with the recommendations of the Ethical Clinical Research Committee (CEIC) of the western health area of Valladolid. The protocol was approved by the CEIC with reference number CEIm PI128-18. All subjects gave written informed consent in accordance with the Declaration of Helsinki.

Fecal content from 57 adults (between 29 and 94 years old, $57 / 43$ male/female ratio) was sampled from November 2016 to April 2018: 51 patients were sampled once, four twice, and two individuals three times. Stool samples were stored at $-80^{\circ} \mathrm{C}$ until use.

Stool samples were initially tested for the presence of both glutamate dehydrogenase $(\mathrm{GDH})$ and toxins $\mathrm{A}$ and $\mathrm{B}$ by the lateral flow assay C.Diff Quik Chek Complete assay (Techlab). The GeneXpert $C$. difficile PCR assay (Cepheid) was used for the detection of the toxin $B$ gene $(t c d B)$, binary toxin, and $t c d C$ deletion that identifies ribotype 027 .

\section{Measurement of Redox Potential}

One gram of feces were diluted in $25 \mathrm{~mL}$ of distilled water and centrifuged at $8000 \mathrm{rpm}$ for $10 \mathrm{~min}$. A pH and redox meter (GLP21 and Hach 5262PCE-228-R) was used to measure the $\mathrm{pH}$ and the redox potential according to the manufacturer's instructions.

\section{Total DNA Extraction}

For each stool sample, $220 \mathrm{mg}$ of feces was homogenized and total DNA extracted using the QIAamp DNA Stool Mini Kit (Qiagen), according to manufacturer's instructions. The DNA concentration was determined using a Qubit ${ }^{\circledR}$ fluorimeter (Invitrogen). A second DNA extraction from some of the samples and subsequent sequencing validated the characterization of the microbiota (data not shown).

\section{S rRNA Gene Amplicon Library Preparation and Sequencing}

Microbial diversity was studied by sequencing the amplified V3V4 region of the $16 \mathrm{~S}$ rRNA gene using previously reported primers and PCR conditions (Klindworth et al., 2013). Sample multiplexing, library purification, and sequencing were carried out as described in the "16S Metagenomic Sequencing Library Preparation" guide by Illumina. Libraries were sequenced on a MiSeq platform at the University of Burgos, leading to 300-bp, paired-end reads.

\section{Bioinformatics and Data Analysis}

Demultiplexed paired-end fastq files were processed using QIIME2 pipeline version 2018.6 (Caporaso et al., 2010) and dada2 (Callahan et al., 2016) and feature-table (McDonald et al., 2012) plugins were used for quality filtering of the reads, merging of the paired ends, chimera removal, and assignation of amplicon sequence variants (ASV). We truncated reverse reads to $240 \mathrm{bp}$ 
using the "-p-trunc-len-r" option implemented in the dada2 plugin due to decreased quality scores of the sequences at the end of the reverse reads. A phylogenetic tree was built using alignment (Katoh and Standley, 2013) and phylogeny (Price et al., 2010) plugins. Alpha and beta diversity analysis were performed using the diversity ${ }^{3}$ and emperor (Vazquez-Baeza et al., 2013) plugins. Samples were rarefied to 17,520 reads per sample for beta-diversity analysis to reduce the bias due to different sequencing depths (only sample MS1498 was excluded from beta-diversity analysis). A pre-trained Naïve Bayes classifier based on the SILVA database (Pruesse et al., 2007), which had been trimmed to harbor the V3-V4 region of the 16S rRNA gene, was applied to assign taxonomy to the ASV using the feature-classifier plugin (Bokulich et al., 2018).

Plotting was carried out in the $\mathrm{R}$ environment ${ }^{4}$, using ggplot 2 (Wickham, 2016) and reshape 2 (Wickham, 2007) packages. Weighted UniFrac distance matrices calculated with QIIME2 were represented as principal coordinates (principal component analysis - PCoA) to compare bacterial community composition based on the relative abundance of ASV. ASV assigned to the family Peptostreptococcaceae were extracted from the feature table and used to construct a heatmap using JColorGrid (Joachimiak et al., 2006).

\section{RESULTS}

\section{Description of the Study Population}

Sixty-five fecal samples of the 57 patients with diarrhea enrolled in this study were studied. Figure 1A summarizes the patient metadata; there were no significant differences between gender, $62 \%$ of the patients were $\geq 65$ years old, $73.7 \%$ were healthcareassociated cases, and 19 patients (33.3\%) died before August 2018. All patients were reported to be CDI positive as they were positive for GDH antigen and the gene encoding toxin $\mathrm{B}$ $(t c d B)$, but negative for the $C$. difficile ribotype 027 test. Some samples were negative for the in situ detection of the toxin in the stool and 14 samples were PCR positive for the binary toxin gene (MS0138, MS0148, MS0151, MS0155, MS0211, MS0212, MS0220, MS0223, MS1497, MS1506, MS1508, MS1509, MS1748, MS1753). Remarkably, patients who tested positive for the binary toxin gene did not show severe diarrhea. At least one previous hospital admission was recorded for all but two patients, prior to CDI during the previous year. The number of previous hospital admissions was higher for patients receiving healthcare in hospital than those receiving community care (Figure 1B). All individuals had received antibiotic treatment prior to developing diarrhea and some had also received proton pump inhibitors, except patient MS0215, who came to the emergency room with severe diarrhea, without previous antibiotic treatment. This patient showed an abnormal relative abundance of the Streptococcus genus (10.95\%) and the Bacteroides abundance was below 50\%. Upon hospital admission, all inpatients were administrated antibiotics after the positive diagnosis of $C$. difficile.

${ }^{3}$ https://github.com/qiime2/q2-diversity

${ }^{4}$ https://www.r-project.org
Antibiotherapy generally consisted of metronidazole and then vancomycin if the diarrhea persisted. Only patient MS1496 was finally successfully treated with fidaxomicin, because of multiple recurrences. The most abundant diarrhea-related bacteria for two patients (MS0141 and MS0227) were Staphylococcus and Klebsiella. Neither co-infection nor these pathogens being the primary cause of diarrhea, instead of $C$. difficile, can be ruled out for these two patients.

\section{Diversity of Bacterial Microbiota in Feces From Individuals With CDI}

A total of $7,474,887$ reads survived the quality filtering process $(108,239 \pm 53,034 \mathrm{reads} / \mathrm{sample})$. This is the first time that QIIME2 has been used to analyze the gut microbiota associated with CDI, and, different from previous studies, sequences are not clustered together into "Operational Taxonomic Units" (OTUs) using a certain dissimilarity threshold (generally 97 or 99\% similarity). Amplicon Sequence Variants (ASVs) were obtained instead and represent much higher taxonomic resolution than OTUs, as single-nucleotide differences over the sequenced gene region are taken into account (Callahan et al., 2017). A total of 3,477 ASVs were identified among all samples. We observed lower bacterial diversity than that obtained using OTUs; between 32 (MS0218) and 352 (MS0138) different bacterial ASVs were identified in each fecal sample, significantly lower than the expected 1,000 OTUs that are estimated to exist in a healthy human gut. This reduction of diversity could be associated with the bacterial dysbiosis linked to CDI. However, the main alpha diversity indices, such as the Chao richness estimator, and the Shannon and Simpson diversity indices were $134.32 \pm 80.6$, $4.01 \pm 1.25$, and $0.86 \pm 0.12$, respectively, indicating that the observed reduced alpha diversity was true, and not a sequencing artifact.

We analyzed beta-diversity between samples by calculating weighted UniFrac distance matrices and representing them as principal coordinates (Figure 2). There were two clusters (A and $\mathrm{B}$ ) that described $38.9 \%$ of the variability in the $\mathrm{x}$-axis. The differences between the two clusters were due to the most abundant bacterial families: Enterobacteriaceae and Enterococcaceae in cluster A, Bacteroidaceae and Lachnospiraceae in cluster B. Samples of only 15 patients were assigned to Cluster A (representing $26.31 \%$ of the patients). However, given that C. difficile could be ruled out as the primary etiological agent for four patients, the percentage dropped to $20.75 \%$. In addition, the PCoA plot showed the points to be more widely dispersed in cluster A than in cluster B (Figure 2). Most of the patients in this cluster were either immunosuppressed (transplant patients) or immunocompromised (cancer patients), with a history of aggressive antibiotherapy. In contrast, cluster B was compact (Figure 2) and contained most of the samples $(79.24 \%$ of the patients), suggesting that this group may represent the general CDI scenario.

The relative abundance of the 15 most abundant families in the fecal samples is shown in Figure 3, in which samples are segregated into clusters A and B from the PCoA. Bacteroidaceae was the most abundant family overall (31.01\%). However, 

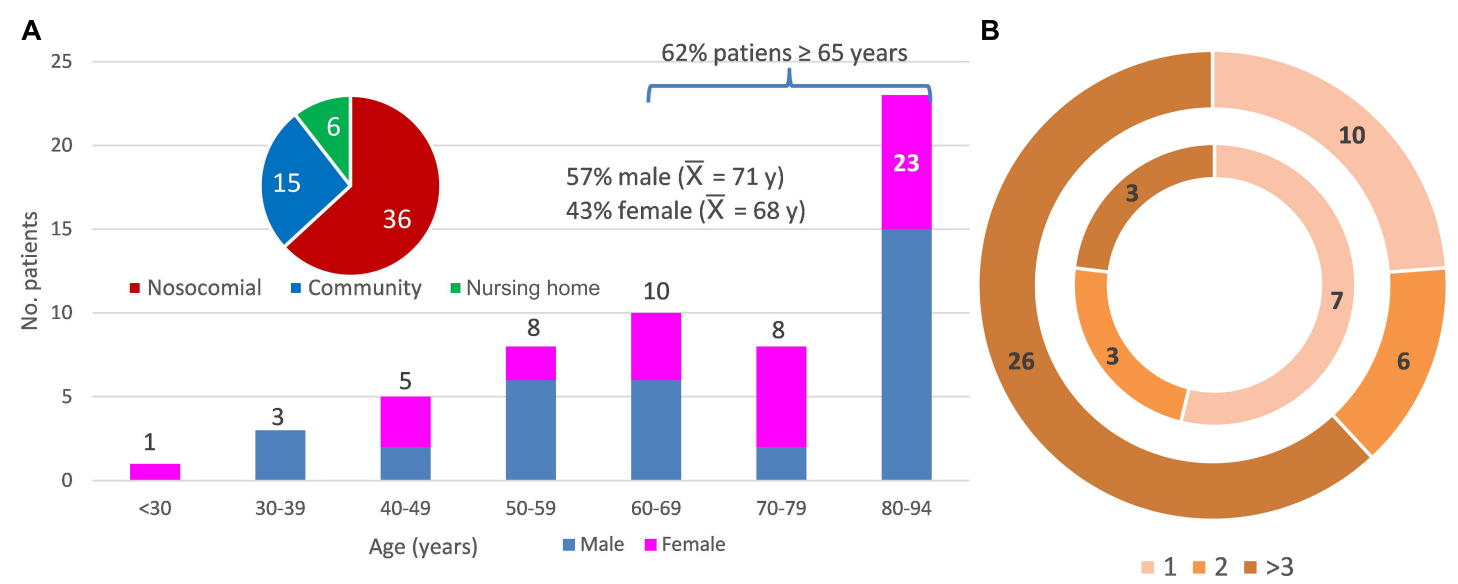

FIGURE 1 | Metadata of the 57 patients studied. (A) Distribution of the patients according to age ( $y$, years and $\bar{X}$, average age), sex (female and male), and community-, healthcare in a nursing home-, and nosocomial-acquired diarrhea. (B) Distribution of the patients according to the number of previous hospital admissions (1, 2, or more than 3 previous hospital admissions). The inner ring indicates a community origin (there were two patients with no previous admission not shown in the figure) and the outer ring, nosocomial or healthcare-in-a-nursing home origin of the infection.

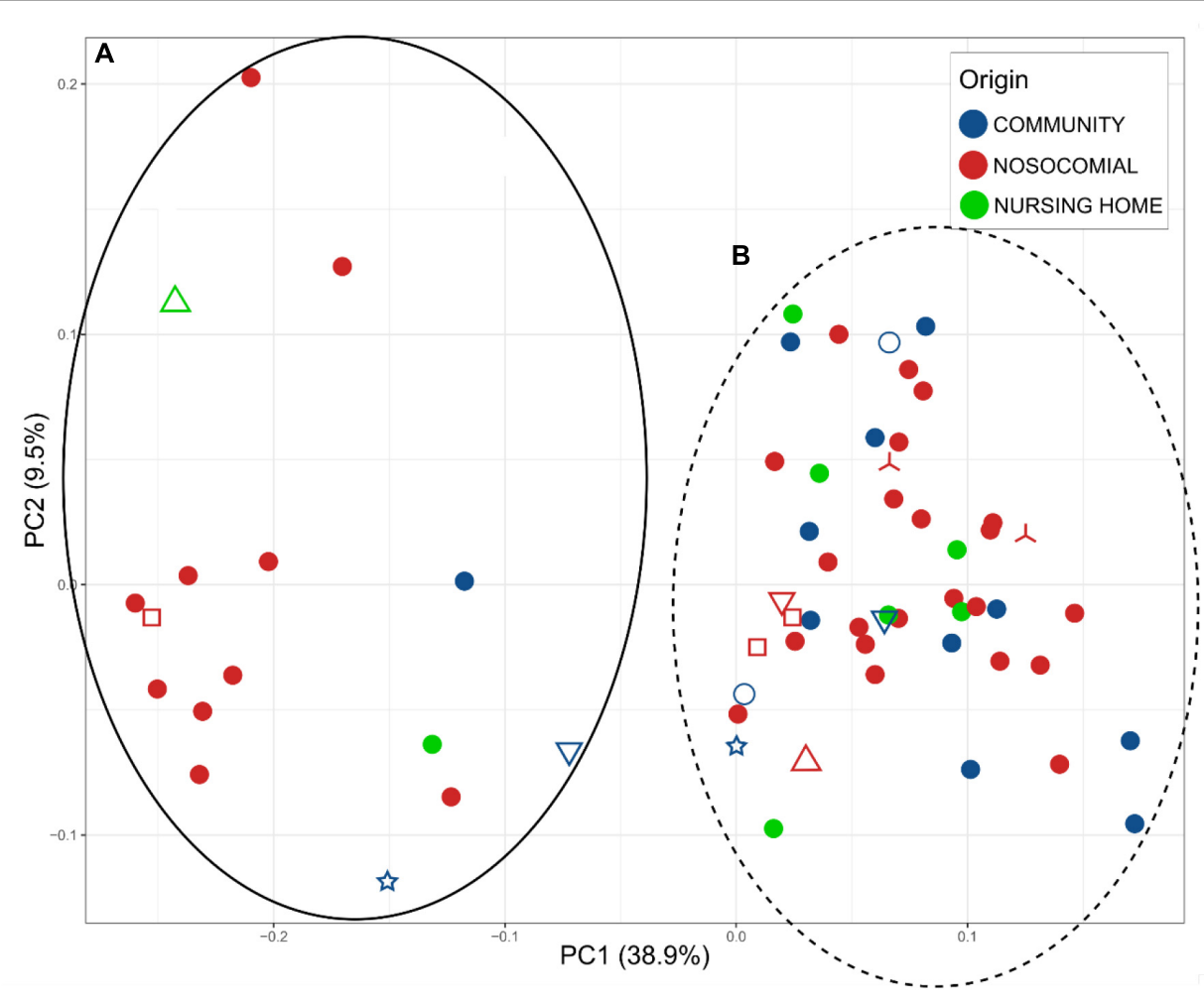

FIGURE 2 | Principal Coordinates Analysis (PCOA), representing the total microbiota of the samples plotted according to the origin of the infection: blue, community; red, nosocomial; and green, nursing home. Two clusters $\mathbf{A}$ (full black) and $\mathbf{B}$ (dot lane) were observed. Different samples from the same individual are indicated by the shapes $\nabla$ MS0217, MS1502, and MS1193; $\square$ MS0209, MS0222, and MS0214; $\triangle$ MS0155 and MS0147; 饵 MS01508 and MS1506; 人 MS0144 and MS0150; and $\bigcirc$ MS1746 and MS1496.

the percentage varied from $0 \%$ in 12 samples to $81.47 \%$ in a sample from a patient who had a dental procedure, was treated with clindamycin, and developed mild diarrhea. Patients who lacked Bacteroidaceae (mainly in Cluster A) had severe disease and were previously treated for diarrhea with an aggressive or prolonged antimicrobial treatment. In contrast, the diarrhea was less severe and the patients who provided samples in which Bacteroidaceae was the most abundant family (mainly Cluster B) recovered easily. Indeed, there was a significant difference in the distribution of Bacteroidaceae 


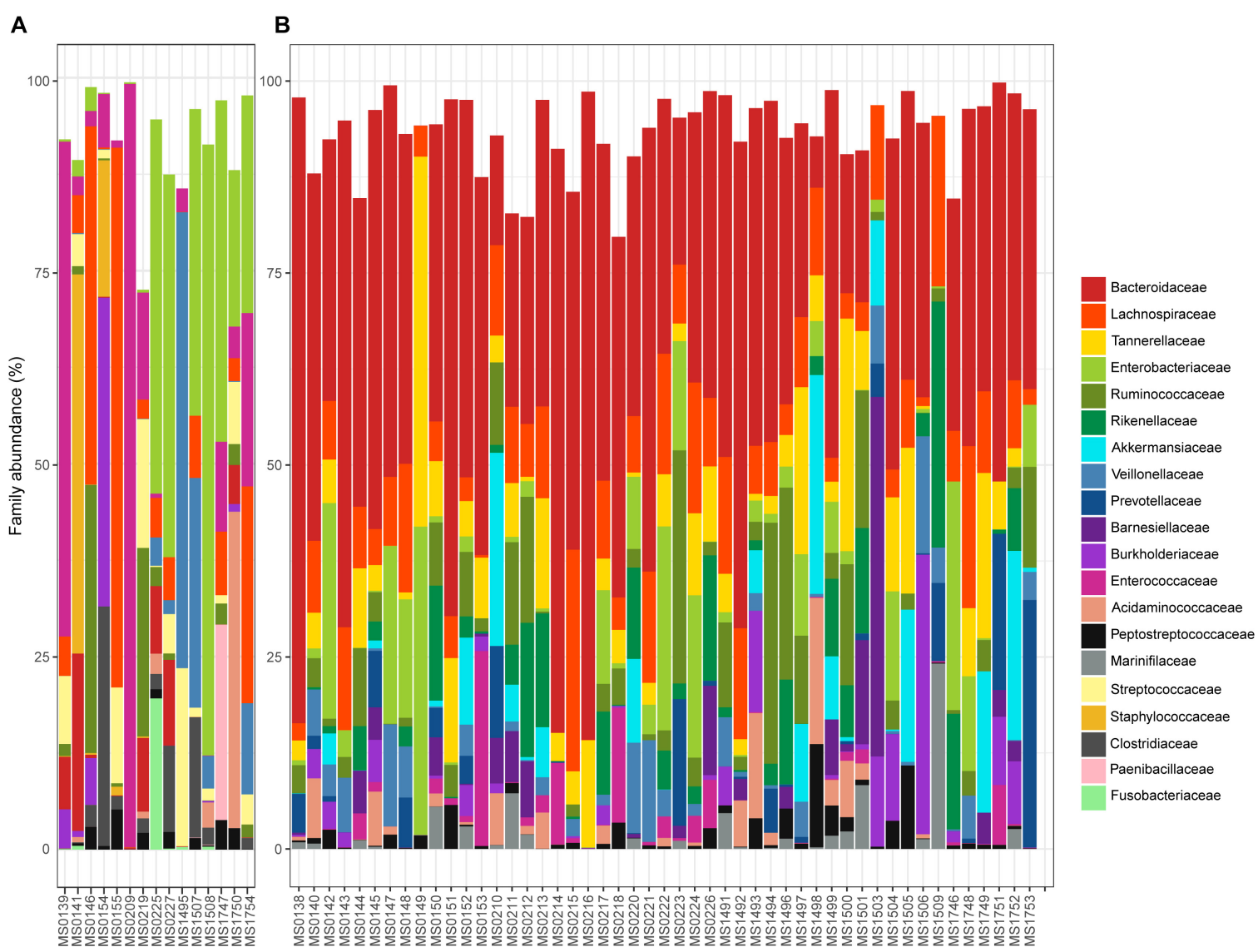

FIGURE 3 | Relative abundance (\%) of the 15 most abundant families found in the samples plotted in the A or B cluster of the PCoA: (A,B), respectively.

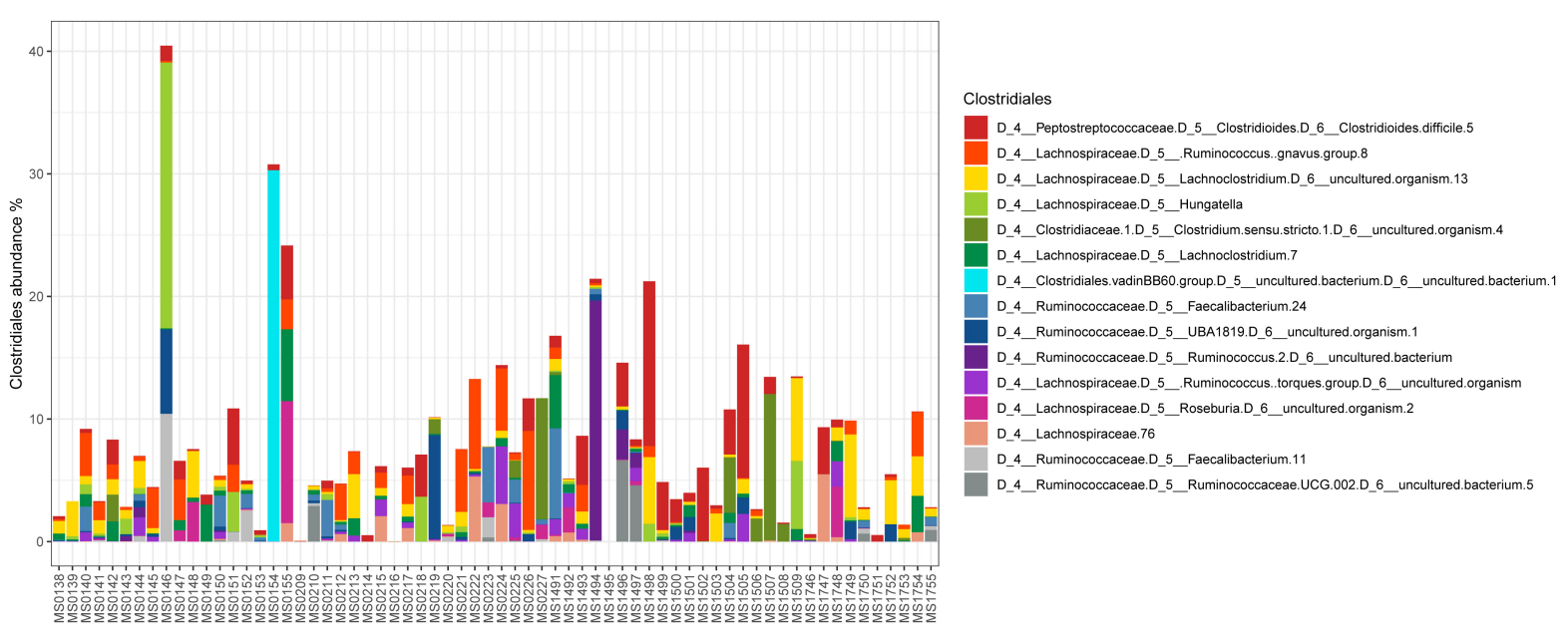

FIGURE 4 | Abundance of taxa within the order Clostridiales.

between cluster A and cluster $\mathrm{B}$; the relative abundance of Bacteroidaceae was very low and even absent in many samples (5.57\% overall, Figure 3A) in cluster A, whereas it was dominant in cluster B, with a relative abundance of $36.91 \%$ (Figure 3B).
Four other bacterial families had an overall relative abundance of over 5\%: Enterobacteriaceae 9.82\%, Lachnospiraceae 9.33\%, Tannerellaceae $6.16 \%$, and Ruminococcaceae 5.64\%. Together with Bacteroidaceae, these five bacterial families represented almost two thirds of the bacterial diversity. Most are obligate 


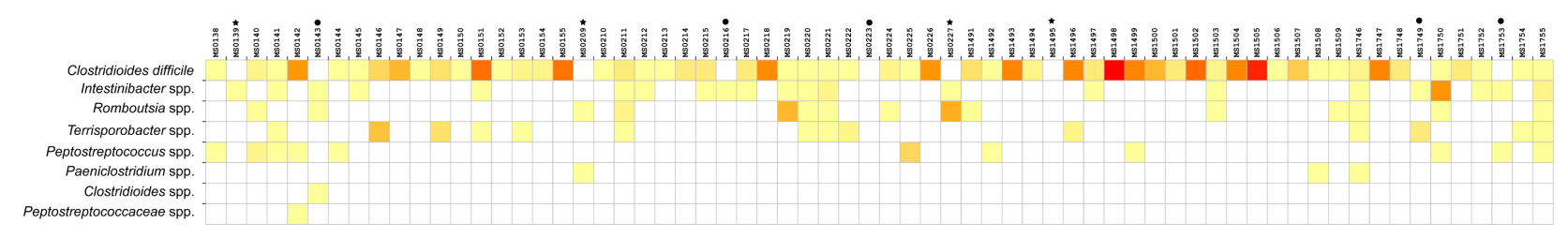

FIGURE 5 | Heat map showing the distribution and relative abundance of members of the Peptostreptococcaceae family grouped (in rows) within the different samples (in columns). C. difficile was absent from nine samples: asterisks indicate the negative samples of cluster A and black dots the negative samples of cluster B.

anaerobes, indicating severe changes in the redox potential that can produce gut bacterial dysbiosis. Differences in the relative abundance of these bacterial families in clusters A and B are shown in Figure 3. Enterobacteriaceae was the most abundant family in cluster A (22.66\% overall, but ranged from $0 \%$ in sample MS1495 to $79.13 \%$ in sample MS1508), whereas the relative abundance of Lachnospiraceae was significantly lower $(4.96 \%$ overall).

Several minority bacterial families (with an overall relative abundance below 5\%), such as Enterococcaceae, Veillonellaceae, Rikenellaceae, Akkermansiaceae, Burkholderiaceae, Acidaminococcaceae, Prevotellaceae, Streptococcaceae, Barnesiellaceae, Peptostreptococcaceae, Staphylococcaceae, Marinifilaceae, and Fusobacteriaceae, were particularly abundant in some samples. For example, the microbiota of sample MS0227, that came from a patient who had bloody diarrhea, fever, and was vomiting, was dominated by Enterobacteriaceae and Pasteurellaceae (49.57 and $9.52 \%$ relative abundance, respectively) and had a low abundance of Bacteroidaceae (11.11\%).

Relative abundance of Peptostreptococcaceae family within the order Clostridiales was comparatively low, $1.56 \%$ overall, and C. difficile was the most abundant species within both the order Clostridiales (Figure 4) and the family Peptostreptococcaceae (Figure 5). C. difficile was identified in almost all samples and was detected in all but five diarrheic samples included in Cluster B (11.90\% of the samples in cluster B), in which other components of the family Peptostreptococcaceae were found (not in sample MS0223). We detected C. difficile in $73.33 \%$ of the samples of cluster A. Interestingly, there was a positive correlation between the increase in the abundance of Akkermansia and that of the Clostridioides genus, as it includes only Clostridioides difficile (Figure 6).

\section{DISCUSSION}

Here, we sought to better understand the microbiota potentially associated with CDI by investigating phylogenetic variation across fecal samples from hospitalized individuals and those living in a community setting with diarrhea and a positive diagnosis of $C$. difficile by high throughput sequencing of the $16 \mathrm{~S}$ ribosomal-RNA- gene amplicons in the Microbiology Laboratory of a tertiary hospital in Spain. Many studies have characterized the baseline gut microbiota in healthy adults, in which $90 \%$ is composed of anaerobes. Individuals can be classified into three "enterotypes" based on their microbiota composition, with a predominance of Bacteroides, Prevotella, or Ruminococcus genera (Arumugam et al., 2011). The individuals in this study clearly belonged to enterotype 1, with a high abundance of Bacteroides (31.01\% overall). Many studies of the human intestinal tract have reported few phyla to be present in the gut; Bacteroidetes and Firmicutes generally dominate, whereas Actinobacteria, Proteobacteria, and Verrucomicrobia, are generally minor constituents. We found a higher average abundance of Bacteroidetes (46.51\% in the samples) than in previous studies, followed by $34.70 \%$ for Firmicutes and $13.49 \%$ for Proteobacteria. However, Firmicutes was more prevalent in 13 samples and Proteobacteria in three.

Culture-based studies suggest that all healthy adults share most of the same gut bacterial species, whereas cultureindependent sequencing studies have revealed vast microbial diversity (more than 1,000 species), that varies highly over time and among the population. A change in microbiota composition and a decrease in the richness of bacterial species within individuals with diarrhea and a positive diagnosis of $C$. difficile is to be expected because, although the gut microbiota stabilizes early in life (during the first 3 years), severe interventions, such as antibiotic administration, or diseases can lead to dysbiosis. Decreased diversity of the fecal microbiome has been reported in recurrent Clostridioides difficile-associated diarrhea. Although one of the main alpha diversity indices, the Shannon index, was significantly higher in our study than that reported in other studies (Staley et al., 2018), the findings of our study are in accordance on that decreased diversity, as we observed reduced bacterial diversity; 3,477 different ASVs were identified with high variability between samples and 29 harbored less than 100 different ASVs. Such a reduction of diversity may be associated with the bacterial dysbiosis linked to CDI. The richness of the microbiota composition was also reduced; although the microbiota composition associated with CDI is still unclear, we observed limited variability and a microbiota characteristic of the presence of $C$. difficile, independent of the severity of the disease. Interestingly, the Shannon index was very low in sample MS209, for which most of the reads were assigned to Enterococcaceae.; this inpatient was immunocompromised and aggressive therapy, including broad-spectrum antimicrobials, corticoids, and antifungal medicines, was used.

Bacteroidaceae was the most abundant family along all samples, followed by Enterobacteriaceae. Several families among the most abundant, such as Lachnospiraceae and Ruminococcaceae, have been previously reported to be enriched 
$1.03 \%$ [Ruminococcus] gnavus group $2.91 \%$ Phascolarctobacterium $5.07 \%$ Escherichia-Shigella 6.61\% Parabacteroides $3.05 \%$ Klebsiella $2.07 \%$ Streptococcus $2.81 \%$ Veillonella $1.03 \%$ Roseburia $1.95 \%$ Lachnoclostridium $1.17 \%$ Lachnospiraceae $3.76 \%$ Akkermansia $1.53 \%$ Clostridioides 1.09\% Acidaminococcus $1.77 \%$ Barnesiella 3.64\% Alistipes $1.21 \%$ Faecalibacterium $1.00 \%$ Fusobacterium $4.65 \%$ Enterococcus $1.06 \%$ Staphylococcus $2.16 \%$ Sutterella
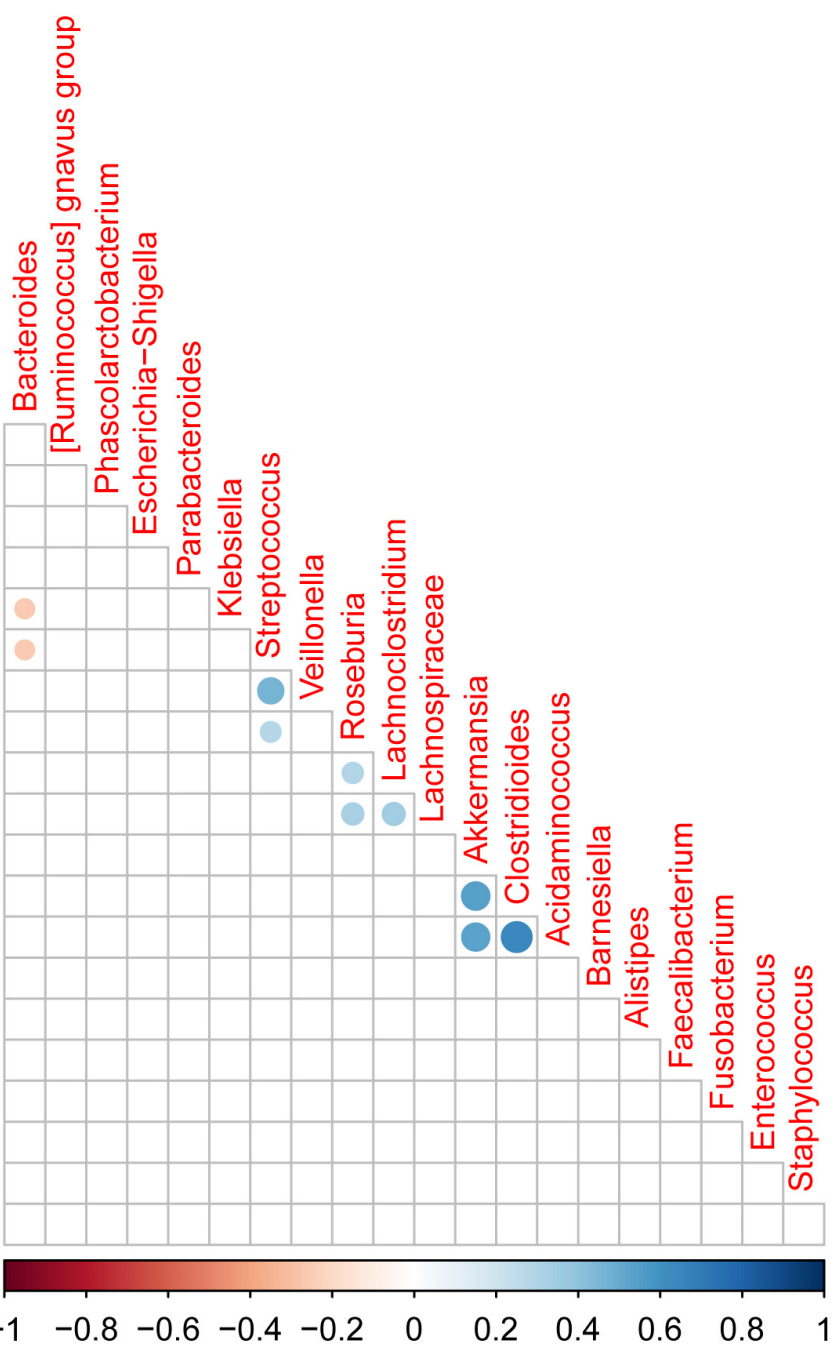

FIGURE 6 | Correlation matrix of the relative abundance of genera above $1 \%$ abundance (significance level $=0.05$ ). The average percentage of each genus among all samples is shown to the left of the name of the genus. A positive correlation was observed between the Akkermansia and Clostridioides genera (C. difficile).

in non-diarrheal cases because they are primary butyrateproducing bacteria in the human gastrointestinal tract and have been associated with the inhibition of $C$. difficile (Schubert et al., 2014). However, the percentage of Bacteroides was lower among the most affected patients and in those with the worst evolution; a reduction in the abundance of this family to below $50 \%$ could be considered to be a marker for worsening of the clinical prognosis. Patients with an abnormally low abundance of Bacteroides $(<10 \%)$ did not recover as well as those with higher percentages. We observed a reduction in the abundance of Bacteroides not only in $C$. difficile associated diarrhea, but also when other pathogenic agents were more abundant. This was true, for example, for samples MS1496 and MS1746, which came from the same patient diagnosed twice, 2 months apart. There was a high abundance of Rikenellaceae (13.65 and 15.05\%), Bacteroidaceae was reduced to approximately $30 \%$, and C. difficile was also identified in the two samples. Thus, the involvement of the pathobiont Rikenellaceae as a triggering factor for the diarrhea cannot be ruled out, as the percentage of $C$. difficile was lower after 2 months.

We identified two clusters in the PCoA (Figure 2); Enterobacteriaceae and Enterococcaceae were the most abundant families in cluster $\mathrm{A}$, whereas Bacteroidaceae and Lachnospiraceae were the most abundant in cluster B. In addition to the different profiles of the bacterial communities, the previous medical interventions, prognoses, and recurrences were different between the two groups. Patients in cluster A were either immunosuppressed (transplant patients) or immunocompromised (cancer patients) and treated with severe antibiotic regimes, including broad spectrum antibiotics. The clinical symptoms within this group included severe diarrhea and an uncertain prognosis, which in many cases was fatal, and the percentage of recurrences higher. Patients in cluster B had had a history of less aggressive antibiotherapy, less severe diarrhea, and only a small incidence of recurrence. The use of aggressive antibiotherapy with broad spectrum antibiotics may have 
reduced the abundance of Bacteroidaceae and Lachnospiraceae in cluster A, giving an advantage to Enterobacteriaceae and Enterococcaceae. The clusters showed a significant difference in the level of $C$. difficile by $16 \mathrm{~S}$ rRNA high throughput sequencing; the percentage of samples negative for C. difficile was $26.67 \%$ for cluster A, whereas it was only $11.90 \%$ for cluster B, for which other members of Peptostreptococcaceae were found in $80 \%$. Thus, the samples belonging to cluster A likely represent a community associated with immune suppression and relatively severe antibiochemoterapy, for which the clinical prognosis of the patients is uncertain and the possibility of recurrence high. Cluster B, consisting of a compact group (Figure 2), and representing most of the samples (79.24\% of the patients) may represent the general CDI scenario.

Differences in microbial composition have been already observed in CDI studies. Sangster et al. (2016) characterized 24 patients, of whom 12 were suspected of having an initial episode of CDI (not recurrent CDI). The Lachnospiraceae, Bacteroidaceae, and Ruminococcaceae families were dominant in both cohorts, but CDI patients showed a predominance of the Peptostreptococcaceae family, with a relative reduction in the abundance of the Bacteroidales and Clostridiales groups, whereas there was a higher abundance of Akkermansia muciniphila and some species of Enterobacteriaceae. Schubert et al. observed that non-diarrheal controls were more likely to have higher levels of several Bacteroidacae, Lachnospiraceae, and Ruminococcaceae families, commonly associated with a healthy microbiome and that Enterococcus species, Enterobacteriaceae, Erysipelotrichaceae, and some Lachnospiraceae families were enriched in some cases (Schubert et al., 2014). However, our findings showed Bacteroidaceae, Lachnospiraceae, and Enterobacteriaceae to be the most abundant families, along with a higher abundance of Enterococcaceae. Segregation of the samples into clusters, showed differences, as Bacteroidaceae and Lachnospiraceae were the most abundant families in cluster B (potentially associated to less severe $\mathrm{CDI}$ ), whereas Enterobacteriaceae and Enterococcaceae were the most abundant in Cluster A, potentially associated with immune suppression, previous use of aggressive antibiotherapy, severe diarrhea, and a high incidence of recurrence.

In a recent study of more than 80 patients, the most abundant bacterial family was Enterobacteriaceae $(>30 \%)$ and the five most abundant families (Enterobacteriaceae, Lachnospiraceae, Bacteroidaceae, Porphyromonadaceae, and Ruminococcaceae) represented less than $50 \%$ of the bacterial diversity (Staley et al., 2018). In our study, Enterobacteriaceae was the most abundant family in cluster A $(22.66 \%$ overall, but ranged from $0 \%$ in sample MS1495 to $79.13 \%$ in sample MS1508), whereas the relative abundance of Lachnospiraceae was significantly lower (4.96\% overall). Staley et al. (2018) also reported similar figures; Enterobacteriaceae was the most abundant bacterial family and the abundance of Lachnospiraceae was also very low. However, Lachnospiraceae was the third most abundant family overall (9.33\%) and the second most abundant family in cluster B, in which its relative abundance was even higher (10.64\%). This finding has been previously reported, but to a significantly lower extent: i.e., 6.5\% (Staley et al., 2018). Although Lachnospiraceae are common inhabitants of the healthy human and mammalian gut microbiota (Lagier et al., 2012), they are highly sensitive to antimicrobial chemotherapy. Thus, a common sign of antibiotic exposure is the reduction or absence of Lachnospiraceae in the gut, creating an environmental niche for opportunistic CDI (Song et al., 2013). Not surprisingly, restoring Lachnospiraceae in infected patients has been shown to help cure C. difficile infections (Song et al., 2013) and it is a bacterial family used in fecal material transplants (Staley et al., 2018). Similarly, a member of the Tannerellaceae family, Parabacteroides distasonis, has been reported to be the most abundant in certain gastrointestinal disorders, such as Crohn's disease (Lopetuso et al., 2018). Sample MS0147 came from a patient with Crohn's disease and the most abundant genus was Escherichia-Shigella at 22.65\%.

Although the relative abundance of Peptostreptococcaceae was comparatively low (1.56\% overall), this family is of prime importance in CDI. The clostridial phylogeny in the phylum Firmicutes has recently been reconsidered and, among other reassignments of the taxa, it has been suggested that $C$. difficile and its close relatives, C. paradoxum and C. sticklandii, be reclassified within the family Peptostreptococcaceae in the order Clostridiales as a tentative solution to resolve various taxonomical problems (Yutin and Galperin, 2013). As expected, C. difficile was the most abundant species within both the order Clostridiales and the family Peptostreptococcaceae, and was identified in almost all samples. We also observed a correlation between the presence of Akkermansia and Peptostreptococcaceae as previously reported (Sangster et al., 2016). Sangster et al. (2016) also reported an increase in the abundance of Akkermansia muciniphila in CDI patients, potentially linked to the capacity of Akkermansia to degrade mucin, which may provide a selective advantage in CDI. In our study, samples MS0223 and MS1753 exhibited an abnormal expansion of the family Prevotellaceae ( $16.50 \%$ and $32.25 \%$, respectively). Prevotellaceae is a family that can enzymatically disrupt mucosal barrier function and tends to be more abundant in intestinal biopsy samples isolated from patients with inflammatory bowel disease (IBD) (NagaoKitamoto et al., 2016).

Predicting microbiota dynamics in individuals and determining whether changes in composition are associated with varying severity and outcomes of CDI will require determining whether such changes lead to the disease. Wilson and Perini (1988) and latter other authors, demonstrated that other microorganisms compete more efficiently than $\mathrm{C}$. difficile for monomeric glucose, $\mathrm{N}$-acetylglucosamine, and sialic acids found in colonic contents, being the reason why $C$. difficile is not a predominant intestinal bacterium. However, when perturbations of the gut microbiota, as occur upon antibiotic administration, intestinal commensal bacteria depletion occur and consequently can help $C$. difficile compete for nutrients to grow, as well as fermentation process decline causing an increase of fecal redox potential. We analyzed the redox potential in fecal content and differs among the samples (minimum $+29.03 \mathrm{mV}$, maximum $+187,67 \mathrm{mV}$, mean \pm standard deviation, $90.7 \mathrm{mV} \pm$ SD35.1). While we tried to establish a correlation between gut redox modification and the microbial alterations observed, it remains unclear, although our findings could 
suggest that modifications of the redox potential might be a key parameter shaping the gut microbiota to be elucidated in future studies. On the other hand, virulence, previous medical treatment, and host immunity will also need to be considered. We analyzed only samples from patients with diarrhea and a positive diagnosis for $C$. difficile. However, several other aspects must be considered in the association of the microbiota to CDI and other gastrointestinal processes. First, a potential drawback of ecological studies of gut microbiota in pathological processes is the misdiagnosis and/or the misassumption of the role of $C$. difficile in the gastrointestinal process, as patients colonized by $C$. difficile can be found for whom the diarrhea is primarily caused by other pathogens, although $C$. difficile is present. That was true in our study for at least four patients who were infected by Klebsiella, Campylobacter, Staphylococcus, or Escherichia. In these cases, co-infection cannot be ruled out, and the differences in the bacterial community profile should be considered with caution. The previous history of antibiotherapy is also highly relevant; the trigger may be dysbiosis caused by prior antibiotic treatment, even in cases in which the gastrointestinal process is caused by $C$. difficile and there is robust evidence for CDI. Similarly, the trigger may be also the status of the immune system. In our study, we were able to segregate the samples into clusters in which these two aspects were highly relevant. Finally, we cannot rule out that the triggering factors were digestive disorders or gastrointestinal diseases (small bowel occlusion, Chron's disease, gastritis, diverticulitis).

In conclusion, our findings show that a reduction in Bacteroides is a clear disadvantage for healthy gut microbiota and can result in a worse CDI prognosis, including severe diarrhea and a high incidence of recurrence. This reduction may be associated with a weakened host immune system and history of aggressive antibiotherapy. In addition, an elevated abundance of

\section{REFERENCES}

Alcalá, L., Reigadas, E., Marín, M., Martín, A., Catalán, P., and Bouza, E. (2015). Impact of clinical awareness and diagnostic tests on the underdiagnosis of Clostridium difficile infection. Eur. J. Clin. Microbiol. Infect. Dis. 34, 1515-1525. doi: 10.1007/s10096-015-2380-3

Arumugam, M., Raes, J., and Bork, P. (2011). Enterotypes of the human gut microbiome. Nature 473, 174-180. doi: 10.1038/nature 09944

Bokulich, N. A., Kaehler, B. D., Rideout, J. R., Dillon, M., Bolyen, E., Knight, R., et al. (2018). Optimizing taxonomic classification of marker-gene amplicon sequences with qiime 2's q2-feature-classifier plugin. Microbiome 6:90. doi: 10.1186/s40168-018-0470-z

Callahan, B. J., McMurdie, P. J., and Holmes, S. P. (2017). Exact sequence variants should replace operational taxonomic units in marker-gene data analysis. ISME J. 11, 2639-2643. doi: 10.1038/ismej.2017.119

Callahan, B. J., McMurdie, P. J., Rosen, M. J., Han, A. W., Johnson, A. J. A., and Holmes, S. P. (2016). DADA2: high-resolution sample inference from Illumina amplicon data. Nat. Methods 13, 581-583. doi: 10.1038/nmeth.3869

Caporaso, J. G., Kuczynski, J., Stombaugh, J., Bittinger, K., Bushman, F. D., Costello, E. K., et al. (2010). QIIME allows analysis of high-throughput community sequencing data. Nat. Methods 7, 335-336.

Davies, K. A., Ashwin, H., Longshaw, C. M., Burns, D. A., Davis, G. L., and Wilcox, M. H. (2016). Diversity of Clostridium difficile PCR ribotypes in Europe: results from the European, multicentre, prospective, biannual, point-prevalence
Akkermansia may be a predictive marker for the presence of a CDI. Finally, a relevant aspect that must be considered in clinical practice is the misdiagnosis of CDI, as patients with a stool sample that tests positive for $C$. difficile are usually diagnosed with CDI and subsequently treated as such. However, co-infection with other pathogenic agents often plays an important role in the development of diarrhea and must be considered when prescribing antibiotic treatment.

\section{Nucleotide Sequence Accession Number}

The 16S rRNA profiling data sequenced in this study were deposited in the Sequence Read Archive of the National Center for Biotechnology Information database under the following study accession number: PRJNA493204.

\section{AUTHOR CONTRIBUTIONS}

MF, MH, and JE designed the study. MF and LL-U performed the diagnosis experiments. $\mathrm{MH}$ performed the sequencing experiments. $\mathrm{MH}$ and NQ performed the bioinformatics analysis and designed the figures. $\mathrm{MH}$ and JE drafted the manuscript. DR-L contributed to the discussion of the results and to the writing of the manuscript.

\section{FUNDING}

This work received financial support of the Gerencia Regional de Salud of Junta de Castilla y León (Spanish Regional Government) (Grant Number GRS 1780/A/18). NMQ received a Ph.D. fellowship from the Spanish National Institute for Agriculture and Food Research and Technology (INIA, Ministerio de Economía, Industria y Competitividad; fellowship FPI2014-020).

study of Clostridium difficile infection in hospitalised patients with diarrhoea (EUCLID), 2012 and 2013. Euro Surveill. 21:30294. doi: 10.2807/1560-7917.ES. 2016.21.29.30294

Davies, K. A., Longshaw, C. M., Davis, G. L., Bouza, E., Barbut, F., Barna, Z., et al. (2014). Underdiagnosis of Clostridium difficile across Europe: the European, multicentre, prospective, biannual, point-prevalence study of Clostridium difficile infection in hospitalised patients with diarrhea (EUCLID). Lancet Infect. Dis. 14, 1208-1219. doi: 10.1016/S1473-3099(14)70991-0

Di Bella, S., Ascenzi, P., Siarakas, S., Petrosillo, N., and di Masi, A. (2016). Clostridium difficile toxins $\mathrm{A}$ and $\mathrm{B}$ : insights into pathogenic properties and extraintestinal effects. Toxins 8:E134. doi: 10.3390/toxins8050134

Hensgens, M. P. M., Goorhuis, A., Dekkers, O. M., van Benthem, B. H. B., and Kuijper, E. J. (2013). All-cause and disease-specific mortality in hospitalized patients with Clostridium difficile infection: a multicenter cohort study. Clin. Infect. Dis. 56, 1108-1116. doi: 10.1093/cid/cis 1209

Joachimiak, M. P., Weisman, J. L., and May, B. (2006). JColorGrid: software for the visualization of biological measurements. BMC Bioinformatics 7:225. doi: 10.1186/1471-2105-7-225

Juul, F. E., Garborg, K., Bretthauer, M., Skudal, H., Øines, M. N., Wiig, H., et al. (2018). Fecal microbiota transplantation for primary Clostridium difficile infection. N. Engl. J. Med. 378, 2535-2536. doi: 10.1056/NEJMc1803103

Katoh, K., and Standley, D. M. (2013). MAFFT multiple sequence alignment software version 7: improvements in performance and usability. Mol. Biol. Evol. 30, 772-780. doi: 10.1093/molbev/mst010 
Klindworth, A., Pruesse, E., Schweer, T., Peplies, J., Quast, C., Horn, M., et al. (2013). Evaluation of general 16S ribosomal RNA gene PCR primers for classical and next-generation sequencing-based diversity studies. Nucleic Acids Res. 41:e1. doi: 10.1093/nar/gks808

Knight, D. R., Elliott, B., Chang, B. J., Perkins, T. T., and Riley, T. V. (2015). Diversity and evolution in the genome of Clostridium difficile. Clin. Microbiol. Rev. 28, 721-741. doi: 10.1128/CMR.00127-14

Lagier, J.-C. (2016). Gut microbiota and Clostridium difficile infections. Hum. Microbiome J. 2, 10-14. doi: 10.1016/j.humic.2016.10.003

Lagier, J.-C., Million, M., Hugon, P., Armougom, F., and Raoult, D. (2012). Human gut microbiota: repertoire and variations. Front. Cell. Infect. Microbiol. 2:136. doi: $10.3389 /$ fcimb. 2012.00136

Lees, E. A., Miyajima, F., Pirmohamed, M., and Carrol, E. D. (2016). The role of Clostridium difficile in the paediatric and neonatal gut - a narrative review. Eur. J. Clin. Microbiol. Infect. Dis. 35, 1047-1057. doi: 10.1007/s10096-016-2639-3

Leffler, D. A., and Lamont, J. T. (2015). Clostridium difficile infection. N. Engl. J. Med. 372, 1539-1548.

Lopetuso, L. R., Petito, V., Graziani, C., Schiavoni, E., Paroni Sterbini, F., Poscia, A., et al. (2018). Gut microbiota in health, diverticular disease, irritable bowel syndrome, and inflammatory bowel diseases: time for microbial marker of gastrointestinal disorders. Dig. Dis. 36, 56-65. doi: 10.1159/000477205

McDonald, D., Price, M. N., Goodrich, J., Nawrocki, E. P., DeSantis, T. Z., Probst, A., et al. (2012). An improved greengenes taxonomy with explicit ranks for ecological and evolutionary analyses of bacteria and archaea. ISME. J. 6, 610-618. doi: 10.1038/ismej.2011.139

McFarland, L. V., Mulligan, M. E., Kwok, R. Y., and Stamm, W. E. (1989). Nosocomial acquisition of Clostridium difficile infection. N. Engl. J. Med. 320, 204-210.

Nagao-Kitamoto, H., Kitamoto, S., Kuffa, P., and Kamada, N. (2016). Pathogenic role of the gut microbiota in gastrointestinal diseases. Intest. Res. 14, 127-138. doi: 10.5217/ir.2016.14.2.127

Price, M. N., Dehal, P. S., and Arkin, A. P. (2010). FastTree 2-approximately maximum-likelihood trees for large alignments. PLoS One 5:e9490. doi: 10. 1371/journal.pone.0009490

Pruesse, E., Quast, C., Knittel, K., Fuchs, B. M., Ludwig, W., Peplies, J., et al. (2007). SILVA: a comprehensive online resource for quality checked and aligned ribosomal RNA sequence data compatible with ARB. Nucleic Acids Res. 35, 7188-7196.

Rupnik, M. (2007). Is Clostridium difficile-associated infection a potentially zoonotic and foodborne disease? Clin. Microbiol. Infect. 13, 457-459.

Sangster, W., Hegarty, J. P., Schieffer, K. M., Wright, J. R., Hackman, J., Toole, D. R., et al. (2016). Bacterial and fungal microbiota changes distinguish $C$. difficile infection from other forms of diarrhea: results of a prospective inpatient study. Front Microbiol. 25:789. doi: 10.3389/fmicb.2016.00789

Schubert, A. M., Rogers, M. A. M., Ring, C., Mogle, J., Petrosino, J. P., Young, V. B., et al. (2014). Microbiome data distinguish patients with Clostridium difficile infection and non-C. difficile-associated diarrhea from healthy controls. mBio 5:e01021-14. doi: 10.1128/mBio.01021-14

Sebaihia, M., Wren, B. W., Mullany, P., Fairweather, N. F., Minton, N., Stabler, R., et al. (2006). The multidrug-resistant human pathogen Clostridium difficile has a highly mobile, mosaic genome. Nat. Genet. 38, 779-786.

Shankar, V., Hamilton, M. J., Khoruts, A., Kilburn, A., Unno, T., Paliy, O., et al. (2014). Species and genus level resolution analysis of gut microbiota in Clostridium difficile patients following fecal microbiota transplantation. Microbiome 2:13. doi: 10.1186/2049-2618-2-13

Smits, W. K., Lyras, D., Lacy, D. B., Wilcox, M. H., and Kuijper, E. J. (2016). Clostridium difficile infection. Nat. Rev. Dis. Primers 7:16020. doi: 10.1038/nrdp. 2016.20

Song, Y., Garg, S., Girotra, M., Maddox, C., von Rosenvinge, E. C., Dutta, A., et al. (2013). Microbiota dynamics in patients treated with fecal microbiota transplantation for recurrent Clostridium difficile infection. PLoS One 8:e81330. doi: 10.1371/journal.pone.008 1330

Staley, C., Kaiser, T., Vaughn, B. P., Graiziger, C. T., Hamilton, M. J., Rehman, T. U., et al. (2018). Predicting recurrence of Clostridium difficile infection following encapsulated fecal microbiota transplantation. Microbiome 6:166. doi: 10.1186/ s40168-018-0549-6

Vazquez-Baeza, Y., Pirrung, M., Gonzalez, A., and Knight, R. (2013). EMPeror: a tool for visualizing high-throughput microbial community data. Gigascience 2:16. doi: 10.1186/2047-217X-2-16

Wickham, H. (2007). Reshaping data with the reshape package. J. Stat. Softw. 21, 1-20. doi: 10.3978/j.issn.2305-5839.2016.01.33

Wickham, H. (2016). ggplot2: Elegant Graphics for Data Analysis. New York, NY: Springer-Verlag.

Wilson, K. H., and Perini, F. (1988). Role of competition for nutrients in suppression of Clostridium difficile by the colonic microflora. Infect. Immun. 56, 2610-2614.

Yutin, N., and Galperin, M. Y. (2013). A genomic update on clostridial phylogeny: Gram-negative spore formers and other misplaced clostridia. Environ. Microbiol. 15, 2631-2641. doi: 10.1111/1462-2920.12173

Conflict of Interest Statement: The authors declare that the research was conducted in the absence of any commercial or financial relationships that could be construed as a potential conflict of interest.

Copyright (c) 2019 Hernández, de Frutos, Rodríguez-Lázaro, López-Urrutia, Quijada and Eiros. This is an open-access article distributed under the terms of the Creative Commons Attribution License (CC BY). The use, distribution or reproduction in other forums is permitted, provided the original author(s) and the copyright owner(s) are credited and that the original publication in this journal is cited, in accordance with accepted academic practice. No use, distribution or reproduction is permitted which does not comply with these terms. 